\title{
Special issue: Symposium on trends in applications of mathematics to mechanics (STAMM) 2008
}

Published online: 27 June 2009

(C) Springer-Verlag 2009

In this special issue of the CMT we collected few extended versions of the general lectures presented at the Symposium on Trends in Applications of Mathematics to Mechanics which took place in Levico (close to Trento, in the Italian Alps) on 22-25 September 2008. The Symposium was organized by P. Colli, I. Mueller, and A. Visintin. The program and further details are available at http://www-dimat.unipv.it/pier/STAMM08files/contributi/. We recall that STAMM is a biennial meeting of the ISIMM (International Society for the Interaction of Mechanics and Mathematics, http://www.isimm.ing.unitn.it/. According to the constitution of the ISIMM : The history of both mechanics and mathematics shows much evidence of the beneficial influence of each of these disciplines on the other. It is the purpose of this Society to promote the interaction of mathematics and mechanics. With this objective, international meetings shall be organized for the presentation and discussion of research at the interface of the two disciplines. As it is also written in the constitution of the ISIMM mechanics should be understood in the broad sense of the word including all relevant physical phenomena.

In full agreement with these major goals of the ISIMM the current Symposium has shown that today the combined use of mathematical modeling and numerical computations produces an increasingly more powerful tool for the understanding and reproduction of reality, and that the symbiosis of mathematics and mechanics has become of paramount importance for science, engineering, medicine and technology-virtually for any human activity. In this spirit the applications of mechanics and mathematics addressed at STAMM 2008 were rather broad and included cosmology, biology, and even traffic dynamics. Mathematical modeling was at the center of the meeting. An incomplete list of problems raised and analyzed by the participants of the meeting includes: multiscale modeling of size effect, component interaction in fluid mixtures, phase-field models, magnetization dynamics, tumor growth, finite-strain plasticity, stress-enhanced diffusion in coal layers, dislocations dynamics, models for binary alloys, stability for reaction-diffusion models, flows on networks and hyperbolic balance laws. Among the diverse expertise of the participants, which is grossly underrepresented by our selection in this volume, the prevailing fields were analysis, theoretical physics, and rational mechanics. The included papers, however, give a good idea of the quality of the invited lectures, that were also complemented by excellent contributed talks and poster presentations.

Pierluigi Colli

Ingo Mueller

Augusto Visintin 